\title{
EXPERIÊNCIAS DE ENFERMEIROS NA EDUCAÇÃO A DISTÂNCIA: UM OLHAR SOBRE AS DIMENSÕES INTERAÇÃO E AUTONOMIA
}

\author{
Ricardo Bezerra Cavalcante ${ }^{1}$, Tarcisio Laerte Gontijo ${ }^{2}$, Lídia Trindade de Castro Silva ${ }^{3}$, \\ Cristiano José da Silva Esteves ${ }^{4}$, Fabricia Almeida Diniz ${ }^{5}$, Daniela Dias Vasconcelos ${ }^{6}$
}

\begin{abstract}
RESUMO: Estudo de caso de abordagem qualitativa que buscou analisar as experiências de enfermeiros na modalidade a distância nas dimensões interação e autonomia. Realizaram-se entrevistas semiestruturadas com 13 enfermeiros de uma Instituição de Ensino Superior em Minas Gerais, entre dezembro de 2014 e fevereiro de 2015. Utilizou-se da Análise de Conteúdo como técnica de análise. Verificou-se que a interação foi destacada por meio dos componentes diálogo e estrutura. O diálogo aconteceu, predominantemente, entre os próprios estudantes e na perspectiva da socialização e do aprendizado colaborativo. Quanto à estrutura, constatou-se a concepção de alta estruturação e o controle favorecidos pela maximização do aparato tecnológico. Na dimensão autonomia, o aprendizado se deu em rede e de forma colaborativa, potencializados pelas características: flexibilidade de horários/tempo/deslocamento, redução de custos e a decisão pela busca do aprimoramento. Conclui-se que a enfermagem tem encontrado na Educação a Distância possibilidades de avanços no conhecimento, entretanto a interação e autonomia necessitam serem maximizadas nestas experiências.
\end{abstract}

DESCRITORES: Educação a distância; Educação em enfermagem; Tecnologia da informação; Educação permanente.

\section{NURSES' EXPERIENCE IN DISTANCE EDUCATION: AN OVERVIEW ON THE DIMENSIONS OF INTERACTION} AND AUTONOMY

\begin{abstract}
This was a case study with a qualitative approach that aimed to analyze nurses' experience in the distance education modality focusing on the dimensions of interaction and autonomy. Semi-structured interviews were carried out with 13 nurses in a Higher Education Institution in Minas Gerais, from December 2014 to February 2015. Content Analysis was used as technique of analysis. It was found that interaction was made possible through the components of dialogue and structure. Dialogue took place mainly among students themselves and in the context of socialization and collaborative learning. Regarding structure, the study found the evidence of high structuring and control made possible by the maximization of the technological apparatus. In the dimension of autonomy learning took place through networking and in a collaborative way, enhanced by the following features: time/displacement flexibility; reduced costs; and the decision to search for improvement. This study found possibilities of advancing knowledge in Distance Education, though interaction and autonomy need to be maximized in these experiences.

DESCRIPTORS: Distance Education; Education in nursing; Information technology; Permanent education.
\end{abstract}

\section{EXPERIENCIAS DE ENFERMEROS CON EDUCACIÓN A DISTANCIA: UNA VISIÓN SOBRE LAS DIMENSIONES INTERACCIÓN Y AUTONOMÍA}

RESUMEN: Estudio de caso, abordaje cualitativo, buscando analizar experiencias de enfermeros en aprendizaje a distancia respecto de dimensiones interacción y autonomía. Se realizaron entrevistas semiestructuradas con 13 enfermeros de Institución de Enseñanza Superior de Minas Gerais, entre diciembre 2014 y febrero 2015. Se aplicó técnica de Análisis de Contenido. La interacción fue destacada por sus componentes diálogo y estructura. El diálogo se dio, fundamentalmente, entre los propios estudiantes, en perspectiva de socialización y aprendizaje colaborativo. Respecto a la estructura, se constató concepción de alta estructuración y de control, favorecidos por maximización del aparato tecnológico. En la dimensión autonomía, el aprendizaje se realizó en red, de manera colaborativa, potenciado por las características: flexibilidad de horarios/tiempo/desplazamiento, reducción de costos y decisión de búsqueda de mejoramiento. Se concluye en que la enfermería encuentra en la Educación a Distancia posibilidades de avances del conocimiento, en tanto interacción y autonomía sean maximizadas en la experiencia.

DESCRIPTORES: Educación a Distancia; Educación en Enfermería; Tecnología de la Información; Educación Continua.

${ }^{1}$ Enfermeiro. Pós-doutor em Ciência da Informação. Docente da Universidade Federal de São João Del Rei. Divinópolis, MG, Brasil.

${ }^{2}$ Enfermeiro. Doutorado em Ciências da Saúde. Docente da Universidade Federal de São João Del Rei. Divinópolis, MG, Brasil. ${ }^{3}$ Discente de Enfermagem. Universidade Federal de São João Del Rei. Divinópolis, MG, Brasil.

${ }^{4}$ Enfermeiro. Mestrando em Enfermagem. Universidade Federal de São João Del Rei. Divinópolis, MG, Brasil.

${ }^{5}$ Enfermeira. Mestre em Enfermagem. Universidade Federal de São João Del Rei. Divinópolis, MG, Brasil.

${ }^{6}$ Enfermeira. Especialista em Saúde da Família. Universidade Federal de São João Del Rei. Divinópolis, MG, Brasil.

Autor Correspondente:

Cristiano José da Silva Esteves

Universidade Federal de São João Del Rei

Av. Sebastião Gonçalves Coelho, 400 - 35501-296 - Divinópolis, MG, Brasil

E-mail: cristianoxkm@gmail.com
Recebido: 18/01/2016

Finalizado: 14/05/2016 


\section{INTRODUÇÃO}

No contexto da saúde, a Educação a Distância (EaD) tem ampliado as possibilidades de educação permanente dos profissionais a partir da disseminação e compartilhamento de conhecimentos dentro e fora do local de trabalho(1).

A Enfermagem também tem utilizado dos recursos da EaD visando à educação permanente de seus profissionais. Isso envolve o desenvolvimento de disciplinas em cursos de pós-graduação, cursos de atualização e aperfeiçoamento em temáticas específicas da área, teleconsultorias, dentre outros ${ }^{(2-5)}$.

Apesar da EaD estar cada vez mais difundida no contexto da enfermagem, é necessário avaliar estas experiências sob a perspectiva dos atores envolvidos, colocando-os no centro do processo avaliativo ${ }^{(6)}$. A avaliação das experiências vivenciadas na EaD podem revelar aspectos que enfatizem suas facilidades, bem como pode apontar avanços necessários para o aperfeiçoamento de sua práxis.

No contexto da Enfermagem, a avaliação realizada por aqueles que estão envolvidos nestas experiências com a EaD, tem revelado contribuições e desafios. Sobre as contribuições, destacam-se a redução de tempo e custos, a flexibilidade e a democratização do acesso e a capilaridade territorial ${ }^{(2,7)}$. Já os desafios que emergem neste cenário apontam a necessidade de adequação dos conteúdos online e os objetos de aprendizagem, a adequação das atividades propostas à realidade cursista e o desenvolvimento de competências tecnológicas ${ }^{(3,5)}$.

Além disso, a interação e a autonomia do indivíduo emergem como grandes desafios a serem compreendidos neste contexto ${ }^{(8)}$. Esses dois últimos aspectos tem ganhado cada vez mais espaço em estudos que defendem a maximização das experiências na $\mathrm{EaD}$, por meio do desenvolvimento da interação entre os vários atores que compõem uma rede virtual, bem como a autonomia neste espaço $^{(8-9)}$. Assim, é preciso desenvolver estudos no contexto da enfermagem que avancem no sentido de compreender a interação e autonomia nas experiências com a EaD. Neste sentido, este estudo tem como questão norteadora: "Nas experiências de enfermeiros na modalidade a distância, como as dimensões, interação e autonomia se desenvolvem?".

Sendo assim, o objetivo deste estudo é compreender as experiências de enfermeiros na modalidade a distância nas dimensões interação e autonomia.

\section{METODOLOGIA}

Trata-se de um estudo de caso de abordagem qualitativa. O uso dessa abordagem justificou-se pela necessidade de enfatizar as pessoas como o foco do processo avaliativo manifestando-se por meio de suas subjetividades ${ }^{(10)}$.

A aplicabilidade do estudo de caso justifica-se por ser um referencial metodológico capaz de captar os acontecimentos contemporâneos e preservar as características holísticas e significativas dos eventos da vida real ${ }^{(11)}$. Neste caso, o evento "unidade de análise" que se investiga são as experiências de enfermeiros com a modalidade a distância nas dimensões interação e autonomia.

Utilizou-se a Teoria da interação a distância como referencial teórico ${ }^{(8)}$. A teoria é composta pelas dimensões "Interação a distância" e "Autonomia do aluno". Na primeira dimensão, há dois componentes que podem ser mensurados: diálogo e estrutura. O diálogo é usado para descrever interações entre os diversos atores (professor, aluno, tutor), podendo ser intencional e valorizado por cada uma das partes. No componente estrutura, trata-se o conjunto de elementos utilizados para a elaboração do curso: apresentação das informações e dos objetivos de aprendizado, temas do conteúdo, estudos de caso, ilustrações, exercícios e avaliações.

A dimensão autonomia envolve as capacidades na tomada de decisões sobre o próprio aprendizado, a flexibilidade no gerenciamento das atividades, a independência para escolhas e a colaboração compartilhada.

Essas dimensões de análise direcionaram a definição das seguintes categorias analíticas: A interação 
a distância: diálogo e estrutura sob a ótica dos enfermeiros; Autonomia dos enfermeiros em suas experiências com a Educação a Distância.

O estudo foi desenvolvido em uma Instituição Federal de Ensino Superior (IFES) de Minas Gerais. $\mathrm{Na}$ instituição há a graduação em Enfermagem (modalidade presencial) que atualmente conta com 360 alunos e 45 professores dos quais 39 são enfermeiros.

Os participantes incluídos neste estudo foram todos os enfermeiros que passaram ou estavam passando por alguma experiência com $\mathrm{EaD}$ na instituição de vínculo profissional e até mesmo em outras instituições. Assim, apesar de assumirem o cargo de docentes na instituição, buscavam por meio da EaD oportunidades de continuidade de seus estudos, o que caracteriza a inserção destes indivíduos em situação de educação permanente.

Realizaram-se entrevistas semiestruturadas com enfermeiros, em seus gabinetes, no período de dezembro de 2014 a fevereiro de 2015, sendo áudio-gravadas e posteriormente transcritas. As entrevistas tiveram uma duração média de 40 minutos. Utilizou-se de um roteiro semi-estruturado contendo as seguintes questões: Fale-me sobre suas experiências com a EaD; Como você poderia avaliar estas experiências em seu processo de ensino-aprendizado?; Fale-me sobre as habilidades tecnológicas que você precisou desenvolver para viabilizar suas experiências.

O convite para as entrevistas foi enviado, inicialmente, via e-mail aos 39 enfermeiros possíveis participantes. Este convite foi enviado duas vezes em um período de duas semanas. Neste convite foi apresentada a proposta de pesquisa, seu objetivo e sua metodologia com os critérios de inclusão. Dos 39 enfermeiros da instituição, 30 responderam ao email alegando que encaixavam nos critérios de inclusão e poderiam participar da pesquisa. Assim, a partir deste rol de 30 possíveis participantes foi realizado sorteio aleatório para a composição da sequência das entrevistas a serem realizadas.

Para a composição do número de entrevistados, utilizou-se o critério de saturação. A saturação de informações é definida como a suspensão da coleta de dados quando os dados obtidos não apresentam mais elementos novos para alcançar o objetivo proposto pelo pesquisador ${ }^{(12)}$. Assim, as entrevistas foram encerradas totalizando 13 enfermeiros. Para manutenção e garantia do anonimato dos participantes, estes foram codificados pela letra "E" seguida do número da entrevista como E1, E2, E3 e assim por diante. Ressalta-se que todos os entrevistados leram e assinaram o Termo de Consentimento Livre e Esclarecido permitindo a realização das entrevistas.

Os dados coletados foram analisados por meio da Análise de Conteúdo modalidade TemáticoCategorial ${ }^{(13)}$. Realizou-se uma "leitura flutuante", que permitiu o primeiro contato com o texto a ser analisado e um alinhamento com os objetivos do estudo. Além disso, foram conjugadas as formulações das hipóteses e a elaboração de indicadores que fundamentaram a interpretação final. Nesta pré-análise fez-se recortes do texto, gerando unidades de registro que foram codificadas e analisadas seguindo as regras da exaustividade, representatividade, homogeneidade e de pertinência. Em seguida realizou-se a abstração de representações do conteúdo. Finalmente, procedeu-se às inferências e interpretações gerando a categorização por convergência das unidades de contexto.

Este estudo foi aprovado pelo Comitê de Ética em Pesquisa com Seres humanos da Universidade Federal de São João Del Rei segundo parecer número 011.

\section{RESULTADOS}

\section{A interação a distância: diálogo e estrutura sob a ótica dos enfermeiros}

A interação emergiu nos discursos dos participantes, primeiramente, a partir dos diálogos e aproximações que contribuíram para a experiência do aprendizado:

[...] a aproximação que a gente vai tendo com este trabalho, essa aproximação, ela é buscadora, a sua vivência no ensino a distância, você acaba aprendendo [...] e você interage, conversa com as outras pessoas que também estão envolvidas, e isso acaba, a gente aprendendo com eles, no cotidiano mesmo. (E9) 
Neste contexto, o diálogo assume outra conformação:

[...] foge da forma tradicional de transmissão de conhecimento via sala de aula, ficar falando, e nós conversamos com vários ao mesmo tempo, inclusive com os tutores e professores [...]. (E6)

Entretanto, os participantes também reconheceram que em alguns momentos o diálogo é prejudicado pela diferença de tempo entre as possíveis fontes de emissão e recepção da informação:

[...] mas você tava com dúvidas, mas ninguém te respondeu naquele tempo... e eu precisei daquela informação naquele momento, mas não tive [...]. (E6)

Esta situação também concorre para o enfraquecimento da interação:

[...] então... acabou que eu fazia muitas coisas sozinho, sem conversar... sem discutir com o tutor ou o professor... isso enfraquece a relação [...]. (E13)

Em relação à estrutura, como um elemento potencilizador da interação, primeiramente, os enfermeiros destacaram a disponibilidade de ferramentas que contribuíram para o desenvolvimento de suas experiências:

[...] acesso a todos os links, que eram disponibilizados, de apoio, tinha links auto explicativos, que você podia ler, reler, várias vezes. Então isso facilitou bastante [...]. (E3)

Outro entrevistado reforça:

[...] o fórum... os vídeos... os chats... é fantástico!!! Eu podia interagir, fazer amizades... isto é algo instigante e te incentiva a continuar estudando [...]. (E13)

No entanto, os entrevistados deixam rastros, em seus relatos, do alto controle que se faz por meio das ferramentas tecnológicas em suas experiências, o que caracteriza a alta estrutura dos cursos:

[...] tem que entregar 7 módulos... e depois bloqueia o sistema, ai depois você não consegue mais fazer [...]. (E6)

Além disso, os entrevistados relataram dificuldades em seguir prazos estabelecidos:

[...] porque você tem até hora pra entregar [...] É a questão do prazo mesmo que dificulta [...]. (E12)

Entretanto, reconheceu-se que as ferramentas disponíveis na plataforma tecnológica, em alguns momentos, não foram capazes de suprir as necessidades de informações individualizadas, gerando um suporte impessoal, frio e inteligível:

[...] o aluno sempre tem o tutorial, mas o tutorial é sempre muito impessoal, ele mostra muito bem o que a plataforma tem, mas ele não consegue identificar as minhas dificuldades [...]. (E1)

Além disso, o aprendizado como uma "tentativa solitária" foi destacado na estrutura disponibilizada nas experiências vivenciadas:

[...] a maioria das coisas a gente aprende mais é sozinho mesmo, é fuçando, é tentando, é quebrando a cabeça e aprendendo com os erros [...]. (E5)

Ainda, outro entrevistado relatou a dificuldade para localizar o que desejava a partir da interface disponibilizada na estrutura do curso:

No começo eu pedalei! Custava achar onde entrava em contato com tal coisa, procurava nos links, perdia tempo... até encontrar [...]. (E10)

Por fim, reconheceu-se a inadequação dos equipamentos usados diante da alta estruturação dos cursos:

[...] muitas vezes o equipamento não tem memória suficiente para aquele curso [...] ele cai a linha toda hora [...] o curso tem muito vídeo, link, material em pdf, muito pesado [...]. (E5) 


\section{Autonomia dos enfermeiros em suas experiências com a Educação a Distância}

Os participantes reconheceram em suas experiências com a EaD a possibilidade de [...] aprimorar seu conhecimento [...]; a flexibilidade de horários [...]. (E4)

Além da redução de custos e de deslocamento:

[...] em casa sem deslocamento, eu acho que isso facilita muito. É sem muito gasto [...]. (E6)

Estas situações, características da modalidade a distância, estariam contribuindo para:

[...] a aprendizagem de uma forma mais autônoma, principalmente por parte do discente, porque ele tem uma liberdade de horário conforme a sua disponibilidade [...]. (E9)

Além disso, o papel do professor assume outra roupagem, bem como a responsabilização do aluno pelo seu aprendizado é um estímulo que se desenvolve:

[...] o professor, realmente eu vi que fazia um papel de ser o facilitador. Então não ficava muito depositado nele... acho que eu sentia mais a responsabilidade em mim, do aprendizado. (E3)

Percebe-se neste contexto um deslocamento do professor para uma postura de mediação e colocase o aluno no centro do processo ensino-aprendizagem.

A autonomia dos entrevistados em suas experiências com a EaD também foi reconhecida a partir das possibilidades de escolhas por diversos cursos, dando continuidade aos estudos, bem como possibilidades de atuação profissional em que se inseriam:

[...] eu comecei com ensino a distância na segunda pós-graduação que eu fiz [...] ai na sequência, eu desenvolvi vários cursos, que eu já perdi até a conta de quantos cursos eu fiz, já fiz mais de um curso ao mesmo tempo, eu escolhi assim [...]. (E5)

Outro entrevistado enfatiza:

[...] eu fiz um curso de pós-graduação, voltada para a área de gestão, a distância [...]. (E10)

De outra forma, outros entrevistados relataram que suas experiências em EaD ocorreram como tutor desta modalidade, em cursos de especialização:

[...] eu tenho experiência enquanto tutor, do curso de especialização de formação de especialistas em saúde da família [...]. (E1)

E também em cursos de atualização:

[...] outra vivência que eu tive como tutor, foi durante a execução de um projeto de extensão em uma outra instituição de ensino superior, em que nós promovemos um curso a distância de atualização pra leigos sobre cuidados com pacientes ostomizados [...]. (E11)

Houve ainda relatos que destacaram o uso da EaD para ministrar disciplinas na graduação em enfermagem, por meio de um portal interativo:

[...] como docente eu tô tendo a experiência com uma disciplina, né, que é da saúde da mulher, do $5^{\circ}$ período que a gente ta tentando integrar um conteúdo, então nós estamos usando o portal [...]. (E5)

Além disso, a realização de recuperações (dependências):

[...] então, na verdade eu trabalhei com ensino a distância em outra instituição, na verdade, lá tinham duas finalidades. Uma eram algumas disciplinas que eram a distância, e a outra, era a dependência [...]. (E7)

Outro entrevistado relatou ter tido experiência com EaD na confecção de material didático, os chamados conteudistas:

[...] foi extremamente valiosa a experiência que eu tive enquanto docente [...] enquanto conteudista também, de material pra ser disponível no ensino a distância [...]. (E9) 
Apesar da autonomia experienciada na modalidade a distância, os enfermeiros apresentaram relatos que demonstram certas dificuldades em exercitá-la. Houve o reconhecimento da inabilidade para a organização do próprio tempo mediante à flexibilidade que a modalidade proporciona:

[...] tá tão na nossa responsabilidade essa organização de tempo... [...] ainda tenho muita dificuldade de as vezes de fazer tudo a tempo e a hora, então a gente acaba deixando pra última semana, para os últimos momentos [...]. (E11)

Por fim, foi apontada certa deficiência a priori no manuseio das ferramentas do ambiente virtual de aprendizado e o reconhecimento de que isto tende a prejudicar o exercício da autonomia:

[...] um ponto dificultador seria realmente a familiarização com as ferramentas de trabalho do computador, que eu vejo que isso pode limitar muito a independência do aluno de EaD para desenvolver um bom curso [...]. (E11)

Neste mesmo sentido, outro entrevistado reforça:

[...] e usar, as ferramentas, né, de forma adequada. [...] se você não conhece a ferramenta, isso fica mais difícil [...]. (E1)

Assim, o conhecimento prévio sobre informática foi reconhecido pelos entrevistados como uma necessidade para o exercício da autonomia em suas experiências na EaD:

[...] primeiro, ter que saber, ter conhecimento de informática. Eu percebi que alguns colegas não tinham tanta habilidade de manuseio de algumas ferramentas de informática, eles tiveram mais dificuldade [...]. (E3)

Outro participante endossa:

[...] como eu já tinha uma facilidade pra trabalhar, né, com ferramentas da informática, eu não senti essa dificuldade de ser independente e realizar o curso. (E12)

\section{- DISCUSSÕES}

Em relação ao diálogo verificamos que o mesmo aconteceu, predominantemente, entre os próprios participantes e na perspectiva da socialização e do aprendizado colaborativo. Nesta conformação de diálogo, rompe-se com a tradicional transmissão da informação/conhecimento unidirecional e estabelecem-se os múltiplos diálogos multidirecionais para dar sustentabilidade às "redes de aprendizagem" (14-15). Assim, a aprendizagem transpõe a distância temporal ou espacial e efetiva-se o encontro, a interação e a cooperação, elementos constitutivos da "presencialidade" que também se manifesta no ciberespaço ${ }^{(16)}$.

Os obstáculos para a realização do diálogo também foram lembrados entre os enfermeiros. Estiveram relacionados à diferença temporal entre os vários participantes nas experiências virtuais. Assim, em algum momento o aprendizado foi solitário ao invés de acontecer de forma solidária e colaborativa. Se por um lado há várias fontes e emissores de informações numa perspectiva colaborativa (solidária) e de compartilhamento, por outro estas fontes/emissores são pessoas que assumem um lugar num determinado tempo e, em algum momento, estes espaços e tempos serão diferentes ${ }^{(15)}$. Isto precisa ser considerado nas experiências em desenvolvimento na enfermagem. O que vai determinar se a interação potencializada pelo diálogo será mais distante ou menos distante é a concepção do curso, seus princípios pedagógicos, suas tecnologias e a relação esperada/motivada entre alunos, professores e tutores ${ }^{(17)}$.

O segundo componente, a estrutura, emergiu dos relatos a partir das ferramentas tecnológicas e na concepção de controle (definição de datas, prazos, limites) presentes nas experiências com a EaD. De forma predominante, os cursos possuem a concepção de alta estruturação, voltados para adultos, proporcionando todo o aparato tecnológico dos ambientes virtuais (fóruns, links, chats, hipertexto, vídeos autoinstrucionais) objetivando o aprendizado de forma controlada e com certo grau de independência. Neste modelo, os elementos disponíveis no curso é que ditam o volume e a frequência de atividades direcionando o ritmo do processo ensino-aprendizado ${ }^{(8)}$. Em cursos com 
alta estruturação tem-se menor diálogo entre professor-aluno, bem como é menor a interação a distância ${ }^{(8)}$. Não podemos avaliar como positiva ou negativa esta conformação, pois isto é uma escolha da instituição ofertante. Entretanto, alertamos para a necessidade de conhecer estas possibilidades e escolher a mais adequada ao público alvo no contexto da educação permanente em enfermagem. Cabe a estas instituições, além dos princípios pedagógicos, discutir o nível de estruturação do curso, as possibilidades e conformações dos diálogos que deverão ser estabelecidos e a aplicabilidade dos conteúdos ao contexto de trabalho que o indivíduo está inserido, neste caso o contexto é o setor da saúde.

Ainda no componente estrutura, os participantes julgaram alguns aspectos como negativos em suas experiências com a EaD. Destacaram que as ferramentas tecnológicas disponibilizadas, em sua maioria, oferecem um suporte impessoal não adequado às demandas individualizadas. Podemos considerar que estes programas de $\mathrm{EaD}$ são construídos, intencionalmente ou não, para o atendimento de uma comunidade onde todos os participantes são iguais, e portanto, têm as mesmas necessidades por informações ${ }^{(18)}$. Estruturar um curso na modalidade a distância, nesta ótica, contradiz as características da própria modalidade em que o próprio enfermeiro se coloca no centro do processo ensinoaprendizado, é ativo neste processo, valoriza-se a sua individualidade, suas demandas e aspirações por conhecimento. Assim, no contexto da Enfermagem, é preciso avançar na criação de ferramentas tecnológicas que valorizem as individualidades e estimulem a formação crítico-reflexiva. Do contrário, suas experiências na EaD serão uma reprodução dos mecanismos tradicionais estabelecidos em uma sala de aula tradicional voltados para a transmissão de conhecimento.

Outra pontuação sobre a estrutura foi a interface inadequada, reconhecida pela dificuldade de localizar no ambiente virtual as informações buscadas. Também é um avanço necessário para a construção de espaços colaborativos no contexto da EaD em Enfermagem. Devido à alta estruturação dos cursos, nas experiências vivenciadas, exige-se dos alunos equipamentos adequados, e na sua impossibilidade inviabiliza-se as experiências de aprendizado. Atualmente, há várias possibilidade de ferramentas tecnológicas no contexto da EaD, porém nem todas ainda são possíveis em equipamentos com baixo poder de processamento. A definição por estas tecnologias, se escolhidas, precisam ser informadas aos pretendentes antes de sua iniciação, pois é uma condição sine qua non para o desenvolvimento pleno do curso.

A dimensão da autonomia foi reconhecida pelos enfermeiros a partir de características presentes nas experiências vivenciadas na modalidade a distância (flexibilidade de horários/tempo/deslocamentos, redução de custos e a decisão pela busca do aprimoramento). Além disso, a relação entre professor e alunos, elemento importante no desenvolvimento da autonomia, encontrou outros personagens e formatos nos relatos dos participantes. Nas experiências destes enfermeiros, os alunos também aprendem com outros alunos, com os professores e também com os tutores, numa relação que responsabiliza o aluno pelo seu próprio aprendizado. Já o professor assume outra roupagem, a do mediador e que também aprende nesta relação. Assim, a autonomia quando exercitada, responsabiliza o próprio aluno pelo seu aprendizado, assim como compartilha esta responsabilidade com outros atores (outros alunos, professores e tutores) ${ }^{(17)}$.

A autonomia, enquanto poder de escolha e decisões sobre suas experiências no contexto da EaD, também apresentou outras roupagens: a possibilidade de assumir vários papéis (docente, discente, tutor) e portanto vivenciá-los; a independência na decisão por vivenciar experiências educacionais variadas e concomitantes também emergiu nos discursos. Na primeira, estabelece-se a possibilidade de reconhecer o lugar do outro, experiênciá-lo e, portanto, identificar neste espaço as dificuldades e possibilidades de ensino-aprendizado. Em uma sociedade com forte apelo ao modelo industrial de ensino, focado na centralização do professor como o centro do processo educacional ${ }^{(19)}$, urge uma nova possibilidade, a de empoderar alunos, docentes e tutores reconhecendo suas potencialidades na construção do conhecimento. Isto para a Enfermagem também é um avanço proporcionado pela modalidade a distância, pois além de promover uma nova configuração do processo ensinoaprendizagem relacionado à profissão, também empodera o enfermeiro para atuar em novas frentes de trabalho: professor, tutor, conteudista, designer e outras estações da cadeia desenvolvimentista de cursos na modalidade a distância.

Em relação à independência na decisão por vivenciar experiências educacionais variadas e concomitantes, demonstra-se primeiramente a possibilidade de continuidade dos estudos como uma 
escolha pessoal. Isso pode ser analisado como um aspecto importante e de autonomia do enfermeiro, pois lhe confere a possibilidade de aperfeiçoamento e posicionamento no mercado de trabalho.

O exercício da autonomia também enfrenta desafios que necessitam serem suplantados no contexto das experiências em EaD na Enfermagem. O primeiro, destacado pelos participantes deste estudo, foi a inabilidade com a própria flexibilização característica da EaD. Na perspectiva destes sujeitos, a organização do próprio tempo e de suas atividades como uma decisão independente é um desafio. A autonomia depende de um processo de maturação que acontece a partir de suas experiências. Assim, os enfermeiros ao entrarem numa comunidade de ideias compartilhadas, quando apoiados por alguém mais competente podem, progressivamente e de forma individualizada (com suas próprias experiências), assumir o próprio aprendizado ${ }^{(8-9)}$.

O segundo desafio para o exercício da autonomia diz respeito à deficiência no que concerne as habilidades tecnológicas prévias. Isto tem sido recorrente no contexto da Enfermagem ${ }^{(20-21)}$. Tal fragilidade pode comprometer o processo de ensino-aprendizagem a partir da EaD.

\section{CONSIDERAÇÕES FINAIS}

A interação foi destacada por meio dos componentes diálogo e estrutura. Em relação ao diálogo, verificou-se que este aconteceu predominantemente entre os próprios estudantes e na perspectiva da socialização e do aprendizado colaborativo. Quanto à estrutura, constatou-se a concepção de alta estruturação e o controle favorecidos pela maximização do aparato tecnológico. Na dimensão autonomia, o aprendizado se deu em rede e de forma colaborativa, potencializados pelas características: flexibilidade de horários/tempo/deslocamento, redução de custos e decisão pela busca do aprimoramento. Porém, desafios como a inabilidade com a própria flexibilização característica da EaD e certa deficiência no que concerne às habilidades tecnológicas prévias, precisam ser superados.

As contribuições deste estudo estão no apontamento de aspectos que contribuem para o desenvolvimento e aperfeiçoamento de cursos voltados à educação permanente de enfermeiros. É preciso potencializar a interação por meio do diálogo, do compartilhamento e do aprendizado cooperativo. A estruturação desses cursos também precisa ser discutida a fim de abarcar o perfil dos profissionais envolvidos nessas experiências. Além disso, a autonomia, como um desafio a ser superado neste contexto, é algo que necessita ser continuamente avaliada com vistas à promover nos cursistas habilidades para o desenvolvimento de suas experiências na EaD. Enfim, espera-se que cursos na modalidade a distância voltados à educação permanente em Enfermagem sejam construídos a partir de uma concepção pedagógica bem delineada, que proporcione interação e estimule a autonomia.

\section{REFERÊNCIAS}

1. Silva AN, dos Santos AMG, Cortez EA, Cordeiro BC. Limites e possibilidades do ensino à distância (EaD) na educação permanente em saúde: revisão integrativa. Ciênc. saúde coletiva. [Internet] 2015; 20(4) [acesso em 07 jan 2016]. Disponível: http://dx.doi.org/10.1590/1413-81232015204.17832013.

2. Holtslander LF, Racine L, Furniss S, Burles M, Turner H. Developing and piloting an online graduate nursing course focused on experiential learning of qualitative research methods. J Nurs Educ. [Internet] 2012; 51(6) [acesso em 07 jan 2016]. Disponível: http://dx.doi.org/10.3928/01484834-20120427-03.

3. Ali WGM. Factors affecting nursing student's satisfaction with e- learning experience in King Khalid University, Saudi Arabia. Int J Learn Develop. [Internet] 2012; 2(2) [acesso em 07 jan 2016]. Disponível: http://dx.doi. org/10.5296/ijld.v2i2.1666.

4. De Souza DC, Diniz SOS, da Silva PS, da Silva TCT, Santiago LC. Educação à Distância: uma metodologia de ensino em expansão na enfermagem. Ver. Rede de Cuidados em Saúde. [Internet] 2013; 7(1) [acesso em 07 jan 2016]. Disponível: http://publicacoes.unigranrio.br/index.php/rcs/article/view/1859.

5. Button D, Harrington A, Belan I. E-learning \& information communication technology (ICT) in nursing education: a review of the literature. Nurse Educ Today. [Internet] 2014; 34(10) [acesso em 07 jan 2016]. Disponível: 
http://dx.doi.org/10.1016/j.nedt.2013.05.002.

6. Patterson BJ, Krouse AM, Roy L. Student outcomes of distance learning in nursing education: an integrative review. Comput Inform Nurs. [Internet] 2012; 30(9) [acesso em 07 jan 2016]. Disponível: http://dx.doi.org/10.1097/ NXN.0b013e3182573ad4.

7. Lahti M, Hätönen H, Välimäki M. Impact of e-learning on nurses' and student nurses knowledge, skills, and satisfaction: a systematic review and meta-analysis. Int. j. nurs. stud. [Internet] 2014; 51(1) [acesso em 07 jan 2016 ]. Disponível: http://dx.doi.org/10.1016/j.ijnurstu.2012.12.017.

8. Moore M, Kearsley G. Educação a Distância - Sistemas de Aprendizagem Online. São Paulo: Cengage Learning; 2013.

9. Moore MG. Theory of transactional distance. In: Keegan D, editor. Theoretical Principles of Distance Education. New York: Routledge; 1993. p. 22-38.

10. Minayo MCS. Análise qualitativa: teoria, passos e fidedignidade. Ciênc. saúde coletiva. [Internet] 2012; 17(3) [acesso em 07 jan 2016]. Disponível: http://dx.doi.org/10.1590/S1413-81232012000300007.

11. Yin RK. Estudo de caso. Planejamento e Métodos. 5ª ed. Porto Alegre: Bookman Editora; 2015.

12. Fontanella BJB, Luchesi BM, Saidel MGB, Ricas J, Turato ER, Melo DG. Amostragem em pesquisas qualitativas: proposta de procedimentos para constatar saturação teórica. Cad. Saúde Pública. [Internet] 2011; 27(2) [acesso em 07 jan 2016]. Disponível: http://dx.doi.org/10.1590/S0102-311X2011000200020.

13. Bardin L. Análise de conteúdo. Lisboa: Edições 70; 2011.

14. Zhu C. Student Satisfaction, Performance, and Knowledge Construction in Collaborative Learning. Educ. technol. soc. [Internet] 2012; 15(1) [acesso em 07 jan 2016]. Disponível: http://www.ifets.info/journals/15_1/12.pdf.

15. Rangel-S ML, Barbosa AO, Riccio NCR, de Souza JS. Redes de aprendizagem colaborativa: contribuição da Educação a Distância no processo de qualificação de gestores do Sistema Único de Saúde - SUS. Interface, Comun., Saúde, Educ. [Internet] 2012; 16(41) [acesso em 07 jan 2016]. Disponível: http://dx.doi.org/10.1590/S141432832012005000031.

16. Backes L, Schlemmer E. Práticas pedagógicas na perspectiva do hibridismo tecnológico digital. Rev. diálogo educ. [Internet] 2013;13(38) [acesso em 07 jan 2016]. Disponível: http://dx.doi.org/10.7213/dialogo.educ.7644.

17. Moore MG. Handbook of Distance Education. $3^{\text {a }}$ ed. New York: Routledge; 2013.

18. Castro MMC, Amorim RMA. A Formação Inicial e a Continuada: diferenças conceituais que legitimam um espaço de formação permanente de vida. Cad. CEDES. [Internet] 2015; 35(95) [acesso em 07 jan 2016]. Disponível: http://dx.doi.org/10.1590/CC0101-32622015146800.

19. Nicoletto SCS, Bueno VRLC, Nunes EFPA, Cordoni Junior L, González AD, Mendonça FF, et al. Desafios na implantação, desenvolvimento e sustentabilidade da Política de Educação Permanente em Saúde no Paraná, Brasil. Saude soc. [Internet] 2013; 22(4) [acesso em 07 jan 2016]. Disponível: http://dx.doi.org/10.1590/S010412902013000400012.

20. Sowan AK, Jenkins LS. Designing, delivering and evaluating a distance learning nursing course responsive to students needs. Int J Med Inform. [Internet] 2013; 82(6) [acesso em 07 jan 2016]. Disponível: http://dx.doi. org/10.1016/j.ijmedinf.2013.02.004.

21. Kobayashi RM, Leite MMJ. As competências tecnológicas no ensino de enfermagem cardiológica. Rev. esc. enferm. USP. [Internet] 2015; 49(6) [acesso em 07 jan 2016]. Disponível: http://dx.doi.org/10.1590/S0080623420150000600014. 